\title{
ESTUDO COMPARATIVO DE MODELOS DE COEFICIENTES DE ATIVIDADE DA FASE LÍQUIDA PARA SEPARAÇÃO DA MISTURA ETANOL-ÁGUA
}

\author{
A. APIO $^{1}$ e N. P. G. SALAU \\ Universidade Federal de Santa Maria, Departamento de Engenharia Química \\ E-mail para contato: ${ }^{1}$ andressaapio@ hotmail.com
}

\begin{abstract}
RESUMO: Neste trabalho foi realizado um estudo comparativo dos resultados alcançados no software EMSO na simulação de modelos de coeficientes de atividade da fase líquida, tais como van Laar, Margules, NRTL e Wilson para a separação da mistura binária etanol-água em relação aos dados experimentais obtidos na operação de uma coluna de destilação contínua em bancada. Esses modelos matemáticos são baseados na energia de Gibbs molar em excesso, como uma função da fração molar de líquido através da qual a relação do coeficiente de atividade é calculada para cada componente do sistema. A fim de avaliar o desempenho dos modelos utilizados, foram realizadas as avaliações de índice Integral de Erro Quadrático, Integral do Erro Absoluto e Integral do Erro Absoluto Ponderado no Tempo. A equação de Wilson, portanto, foi a mais adequada para reproduzir as condições ideais do processo, uma vez que essa frequentemente possui um desempenho superior para misturas de caráter polar-apolar, conforme é o caso.
\end{abstract}

\section{INTRODUÇÃO}

Segundo Bai et al. (2008), o etanol é considerado um dos mais importantes combustíveis renováveis devido aos benefícios econômicos e ambientais do seu uso, sendo uma boa alternativa para substituir o petróleo. Para a sua produção, são realizadas três etapas: (1) obtenção de uma solução de açúcares fermentescíveis; (2) fermentação de açúcares em etanol, (3) separação e purificação do etanol, geralmente por destilação para o caso do etanol hidratado. Além disso, estudos recentes têm sido focados na previsão da ocorrência da mistura azeotrópica, como é o caso da mistura binária etanol-água, e, também no projeto de colunas de destilação com o objetivo de produzir uma separação ótima em termos de especificação de produto, mínimo uso de solvente e, consequentemente, redução de custos de operação.

Nesse quesito, para Kumar et al. (2010), na modelagem da destilação de uma mistura etanol-água, a relação de equilíbrio entre a fase líquida e a vapor é, provavelmente, a etapa mais importante. Esse fato se dá devido ao comportamento não ideal desta mistura, dependendo de 
modelos termodinâmicos para estimar as composições em cada fase, para assim prever o azeótropo da mistura formado na concentração de etanol.

\section{MODELOS DE COEFICIENTES DE ATIVIDADE}

A fim de calcular o coeficiente de atividade da fase líquida, usualmente são empregados modelos derivados de expressões dadas para a energia livre em excesso $\mathrm{G}^{\mathrm{E}}$. Em dada temperatura, a energia livre de Gibbs em excesso é função da composição do sistema, e em menor grau, da pressão, sendo que para pressões baixas e moderadas a dependência da pressão pode ser desprezada. Por conseguinte, os modelos adotados para representação do coeficiente de atividade da fase líquida ficam em função da temperatura e composição do sistema. Esses modelos levam em conta a energia de interação entre as moléculas, expressa na forma de parâmetros binários, bem como a organização das mesmas na mistura.

\subsection{Modelos para a Energia de Gibbs em Excesso Empíricos}

As equações de Margules e as equações de van Laar são casos particulares de um tratamento geral baseado em funções racionais, isto é, em equações para $G^{E} / x_{1} x_{2} R T$ dadas por razões de polinômios. Elas oferecem grande flexibilidade no ajuste de dados do ELV para sistemas binários. Além disso, elas possuem fundamentação teórica limitada e não incorporam uma dependência explícita dos parâmetros em relação à temperatura (ABBOTT et al., 2000).

Os coeficientes de atividade que correspondem a equação de Margules são dados pelas equações 1 e 2 :

$$
\begin{aligned}
& \ln \gamma_{1}=\left[\bar{A}_{12}+2\left(\bar{A}_{21}-\bar{A}_{12}\right) x_{1}\right] x_{2}^{2} \\
& \ln \gamma_{2}=\left[\bar{A}_{21}+2\left(\bar{A}_{12}-\bar{A}_{21}\right) x_{2}\right] x_{1}^{2}
\end{aligned}
$$

Onde os parâmetros ajustáveis são dados por Gmehling (1990):

$$
\bar{A}_{12}=1.6022 \text { e } \bar{A}_{21}=0.7947
$$

Já os coeficientes de atividade que correspondem a equação de van Laar são dados pelas equações 3 e 4:

$$
\begin{aligned}
& \ln \gamma_{1}=A_{12}\left(\frac{A_{21} x_{2}}{A_{12} x_{1}+A_{21} x_{2}}\right)^{2} \\
& \ln \gamma_{2}=A_{21}\left(\frac{A_{12} x_{1}}{A_{12} x_{1}+A_{21} x_{2}}\right)^{2}
\end{aligned}
$$

Os parâmetros ajustáveis são dados por Gmehling (1990): 


$$
A_{12}=1.6798 \text { e } A_{21}=0.9227
$$

\subsection{Modelos para a Energia de Gibbs em Excesso de Composição Local}

O conceito de composição local possibilitou desenvolvimentos teóricos na termodinâmica molecular do comportamento de soluções líquidas. As composições locais, no interior de uma solução líquida, diferentes da composição global da mistura, são supostamente responsáveis pelas orientações moleculares não-aleatórias e interações de curto alcance, que resultam de diferenças no tamanho molecular e nas forças intermoleculares. Esse conceito de modelo para o comportamento de soluções foi introduzido na equação de Wilson, e posteriormente, surgiu o modelo de composição local alterativo NRTL.

Os coeficientes de atividade que correspondem a equação de Wilson são dados pelas equações 5 e 6 :

$$
\begin{aligned}
& \ln \gamma_{1}=-\ln \left(x_{1}+\Lambda_{12} x_{2}\right)+x_{2}\left(\frac{\Lambda_{12}}{x_{1}+\Lambda_{12} x_{2}}-\frac{\Lambda_{21}}{\Lambda_{21} x_{1}+x_{2}}\right) \\
& \ln \gamma_{2}=-\ln \left(x_{1}+\Lambda_{12} x_{2}\right)+x_{2}\left(\frac{\Lambda_{12}}{x_{1}+\Lambda_{12} x_{2}}-\frac{\Lambda_{21}}{\Lambda_{21} x_{1}+x_{2}}\right)
\end{aligned}
$$

Na qual a dependência dos parâmetros para com a temperatura é dada pelas equações 7 e 8 :

$$
\begin{aligned}
& \Lambda_{12}=\frac{v_{2}^{L}}{v_{1}^{L}} \exp \left(\frac{-b_{12}}{R T}\right) \\
& \Lambda_{12}=\frac{v_{1}^{L}}{v_{2}^{L}} \exp \left(\frac{-b_{21}}{R T}\right)
\end{aligned}
$$

Os parâmetros ajustáveis são dados por Gmehling (1990):

$$
\begin{aligned}
& v_{1}^{L}=58.68 \mathrm{~cm}^{3} / \mathrm{g} . \mathrm{mol} \text { e } v_{2}^{L}=18.07 \mathrm{~cm}^{3} / \mathrm{g} . \mathrm{mol} \\
& b_{12}=325.0757 \mathrm{cal} / \mathrm{mol} \text { e } b_{21}=953.2792 \mathrm{cal} / \mathrm{mol}
\end{aligned}
$$

Os coeficientes de atividade que correspondem a equação de NRTL são dados pelas equações 9 e 10:

$$
\begin{aligned}
& \ln \gamma_{1}=x_{2}^{2}\left[\tau_{21}\left(\frac{G_{21}}{x_{1}+x_{2} G_{21}}\right)^{2}+\frac{\tau_{12} G_{12}}{\left(x_{2}+x_{1} G_{12}\right)^{2}}\right] \\
& \ln \gamma_{2}=x_{1}^{2}\left[\tau_{12}\left(\frac{G_{12}}{x_{2}+x_{1} G_{12}}\right)^{2}+\frac{\tau_{21} G_{21}}{\left(x_{1}+x_{2} G_{21}\right)^{2}}\right]
\end{aligned}
$$


Onde:

$$
\begin{aligned}
\tau_{12} & =\frac{b_{12}}{R T} \quad \text { e } \quad \tau_{12}=\frac{b_{21}}{R T} \\
G_{12} & =\exp \left(-\alpha_{12} \tau_{12}\right) \quad \text { e } \quad G_{21}=\exp \left(-\alpha_{12} \tau_{21}\right)
\end{aligned}
$$

Cujos parâmetros ajustáveis são dados por Gmehling (1990):

$$
\begin{aligned}
& \alpha_{12}=0.0417 \\
& b_{12}=-9868.839 \mathrm{~J} / \mathrm{mol} \mathrm{e} b_{21}=15937.565 \mathrm{~J} / \mathrm{mol}
\end{aligned}
$$

\section{CRITÉRIOS DE COMPARAÇÃO}

O desempenho das equações empregadas pode ser determinado a partir da visualização gráfica dos resultados gerados pelas simulações. No entanto, esse tipo de comparação é muito subjetivo, tornando necessária a definição dos índices capazes de monitorar o desempenho dos modelos utilizados. Os critérios de comparação baseados na integral do erro são bastante simples e versáteis, uma vez que consideram todo o comportamento dinâmico do processo e não características isoladas.

No presente trabalho são utilizados três critérios de comparação baseados na integral do erro; integral do erro absoluto (IAE), integral do erro quadrático (ISE) e integral do erro absoluto ponderado no tempo (ITAE); para avaliar o desempenho do controlador para cada método de sintonia testado.

A integral do erro absoluto é a integral do valor absoluto do sinal de erro no tempo. É equivalente a soma das áreas acima e abaixo do valor de referência (MARLIN, 1995), dada pela equação 11.

$$
I A E=\int_{0}^{\infty}|e(t)| d t
$$

A integral do erro quadrático (ISE) é a integral do quadrado do sinal de erro no tempo. Esse indicador penaliza mais valores maiores do sinal de erro (MARLIN, 1995), dada pela equação 12.

$$
I S E=\int_{0}^{\infty}|e(t)|^{2} d t
$$

A integral do erro absoluto ponderado no tempo (ITAE) é a integral do tempo multiplicado pelo valor absoluto do sinal de erro no tempo e penaliza erros que se mantém no tempo (MARLIN, 1995), dada pela equação 13. 


$$
I T A E=\int_{0}^{\infty} t \cdot|e(t)| d t
$$

\section{RESULTADOS E DISCUSSÕES}

Para comparar os modelos de coeficiente de atividade para a fase líquida, escolheu-se por utilizar um modelo para destilação contínua para a simulação dos resultados através do software EMSO. Esse modelo foi previamente validado para uma coluna de destilação em bancada, possibilitando comparar o resultado das simulações utilizando diferentes equações termodinâmicas com os dados experimentais obtidos através da operação desse equipamento no regime contínuo.

Nesse caso, a comparação dos resultados é realizada quando o regime estacionário é alcançado, tendo em vista que na destilação contínua a mesma alimentação e as variáveis de operação são mantidas fazendo com que os estágios de equilíbrio operem nas mesmas condições de temperatura, composição e pressão ao longo da destilação.

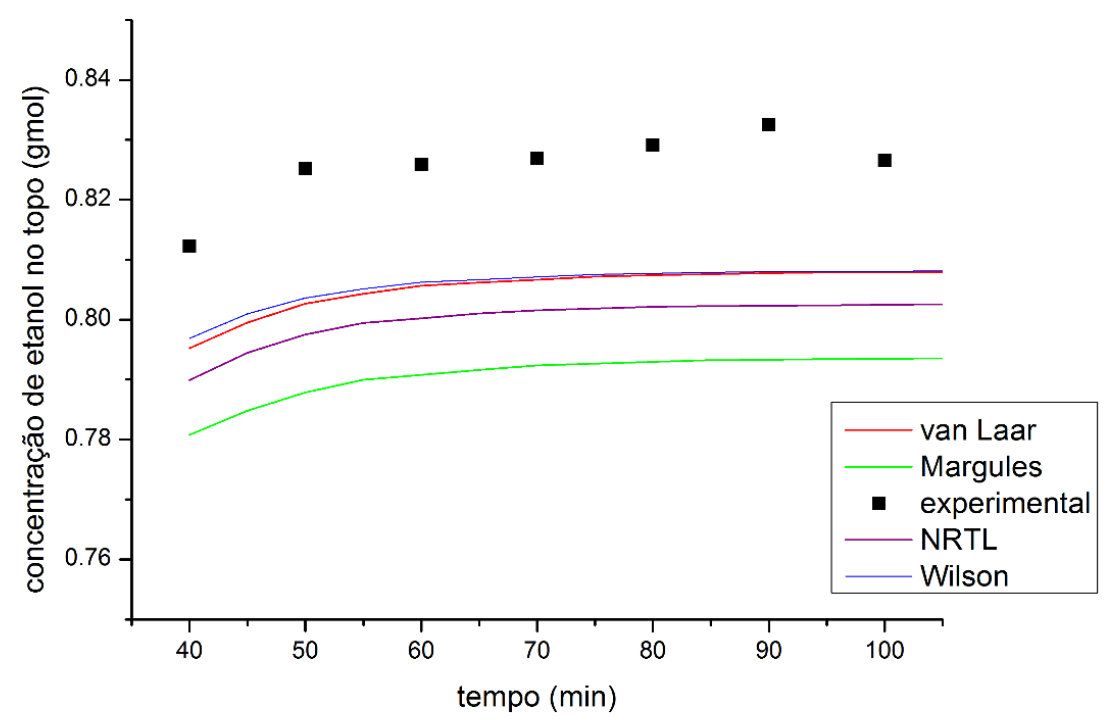

Figura 1 - Comparação entre os resultados de concentração de etanol obtidos através da simulação utilizando diferentes equações termodinâmicas em relação aos dados experimentais.

Para avaliar a melhor abordagem para o cálculo de equilíbrio de fases da mistura binária etanol-água utilizando o modelo em estudo, primeiramente foi utilizada a composição correspondente ao azeótropo da mistura como critério de comparação, conforme visualizado na Figura 1. Observa-se que os modelos de Wilson e van Laar são os que mais se aproximam dos 
valores experimentais. No entanto a análise gráfica não é o suficiente para determinar o modelo mais adequado, sendo necessária a comparação do desempenho através dos critérios da integral do erro, cujo os resultados são apresentados na Tabela 1.

Tabela 1 - Resultado dos critérios de comparação para as diferentes equações termodinâmicas em relação à concentração de etanol.

\begin{tabular}{|c|c|c|c|}
\hline $\begin{array}{c}\text { Equação } \\
\text { termodinâmica }\end{array}$ & IAE & ISE & ITAE \\
\hline van Laar & 1.2719 & 0.0272 & 89.8570 \\
\hline Margules & 2.1442 & 0.0769 & 150.7870 \\
\hline NRTL & 1.5893 & 0.0424 & 112.1300 \\
\hline Wilson & 1.2379 & 0.0259 & 87.8670 \\
\hline
\end{tabular}

A equação termodinâmica de Wilson foi a que apresentou o menor valor nos critérios utilizados, indicando melhor desempenho do que as demais equações.

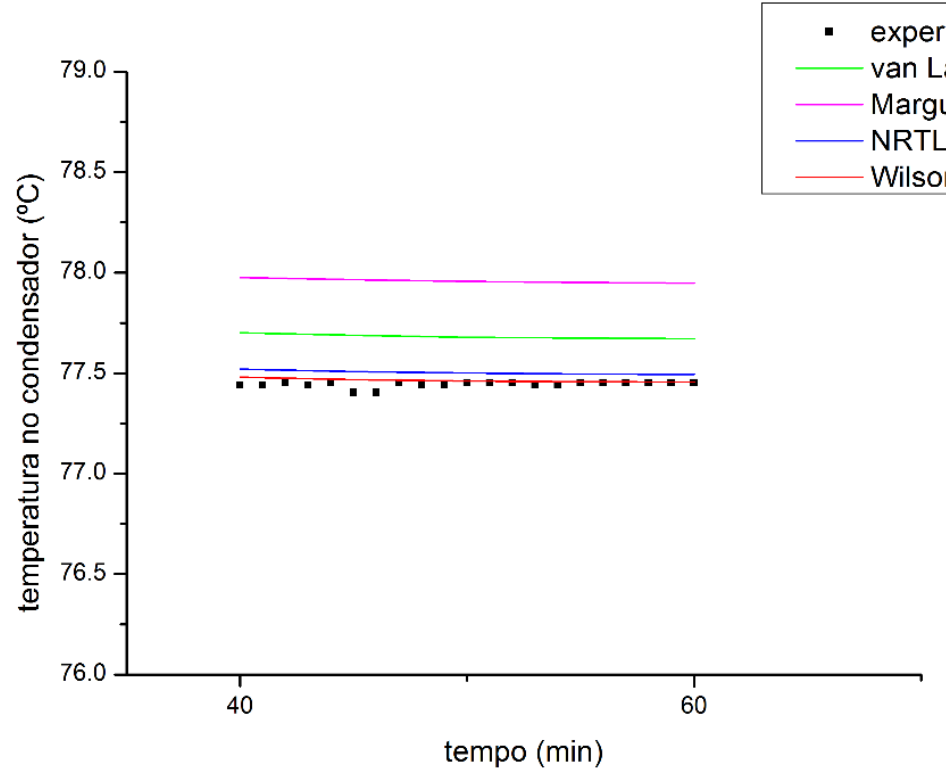

Figura 2 - Comparação entre os resultados da temperatura no condensador obtidos através da simulação utilizando diferentes equações termodinâmicas em relação aos dados experimentais.

Desta vez, como critério de comparação foi utilizada a temperatura no condensador, conforme apresentado na Figura 2, no qual se percebe claramente que o modelo de Wilson é o 
que melhor se ajusta aos dados experimentais. Portanto, para ratificar essa comparação são utilizados os critérios da integral do erro para verificar qual equação possui melhor desempenho, onde os resultados são mostrados na Tabela 2.

Tabela 2 - Resultado dos critérios de comparação para as diferentes equações termodinâmicas em relação à temperatura no condensador.

\begin{tabular}{|c|c|c|c|}
\hline $\begin{array}{c}\text { Equação } \\
\text { termodinâmica }\end{array}$ & IAE & ISE & ITAE \\
\hline van Laar & 4.8635 & 1.1967 & 240.5900 \\
\hline Margules & 10.3973 & 5.4191 & 517.3075 \\
\hline NRTL & 1.2823 & 0.0953 & 61.6675 \\
\hline Wilson & 0.5018 & 0.0252 & 22.7025 \\
\hline
\end{tabular}

Mais uma vez a equação de Wilson obteve os menores resultados para o critério empregado, indicando melhor desempenho em relação às outras equações termodinâmicas.

\section{CONCLUSÃO}

O estudo comparativo dos resultados adquiridos através da simulação dos modelos de coeficiente de atividade da fase líquida com os dados experimentais obtidos na operação de uma coluna de destilação em bancada demonstrou que o modelo de Wilson é o que melhor se ajusta às condições reais do processo. Para o cálculo de equilíbrio de fases da mistura binária etanol-água, foram avaliadas as composições e as temperaturas simuladas com os dados experimentais resultantes da operação da coluna de destilação. Nesses dois critérios, tanto a análise gráfica como o cálculo do erro através da integral do erro demonstraram que o melhor desempenho do modelo de Wilson em relação às demais equações.

A equação de Wilson é, portanto, um modelo de composição local, no qual a sua base teórica está relacionada a sua termodinâmica molecular. Além disso, seus parâmetros são funções explícitas da temperatura e seu desempenho é superior para misturas de caráter polar-apolar, conforme é o caso da mistura binária etanol-água. Essas vantagens tornaram o uso desse modelo adequado para o estudo em questão.

\section{REFERÊNCIAS}

ABBOTT, M.M., SMITH, J.M., VAN NESS, H.C. Introdução à Termodinâmica da Engenharia Química. Editora LTC, Quinta Edição, Rio de Janeiro, Brasil, 2000.

BAI F.W.; ANDERSON, W.A.; MOO-YOUNG, M. Ethanol fermentation technologies from sugar and starch feedstocks. Biotechnology Advances, 25, p. 89-105, 2008. 
GMEHLING J., ONKEN U., AND ARLT W. Vapor-Liquid Equilibrium Data Collection. Chemistry Data Series, Volume I, parts 1-8, 1974-1990

MARLIN, T.E. Process Control. New York: McGraw-Hill, 1995.

KUMAR, S.; SINGH, N.; PRASAD, R. Anhydrous ethanol: A renewable source of energy. Renewable and Sustainable Energy Reviews 14, o. 1830-1844, 2010. 